\title{
BMJ Open Health risk behaviours among migrants by duration of residence: protocol for a systematic review and meta-analysis
}

\author{
Lisa Berg (D) ,1,2 Nina-Katri Gustafsson,, ${ }^{1,2}$ Helena Honkaniemi, ${ }^{1,2}$ Sol Pia Juárez ${ }^{1,2}$
}

To cite: Berg L, Gustafsson N-K, Honkaniemi $\mathrm{H}$, et al. Health risk behaviours among migrants by duration of residence: protocol for a systematic review and meta-analysis. BMJ Open 2020;10:e038388. doi:10.1136/ bmjopen-2020-038388

- Prepublication history and additional material for this paper is available online. To view these files, please visit the journal online (http://dx.doi.org/10. 1136/bmjopen-2020-038388).

Received 09 March 2020 Revised 01 July 2020 Accepted 23 September 2020

Check for updates

(c) Author(s) (or their employer(s)) 2020. Re-use permitted under CC BY-NC. No commercial re-use. See rights and permissions. Published by BMJ.

${ }^{1}$ Department of Public Health Sciences, Stockholm University, Stockholm, Sweden

${ }^{2}$ Centre for Health Equity Studies, Stockholm University/ Karolinska Institutet, Stockholm, Sweden

Correspondence to

Dr Lisa Berg; lisa.berg@su.se

\section{ABSTRACT}

Introduction International migrants' health has often been found to deteriorate in new countries, partly due to changes in health risk behaviours such as alcohol consumption, tobacco use, physical inactivity, and poor dietary habits. However, limited efforts have been made to comprehensively evaluate the extent to which migrants adopt unhealthy risk behaviours with longer duration of residence. This systematic review and meta-analysis will summarise evidence on international migrants' behavioural patterns by duration of residence in multiple country contexts.

Methods and analysis PubMed/MEDLINE, Web of Science and ProQuest databases will be searched for quantitative or mixed-method observational studies published in peer-reviewed scientific journals between 1 January 2000 and 31 December 2019. Studies comparing foreign-born individuals by duration of residence will be included. Information on study characteristics, descriptive statistics and measures of effect will be extracted. All included studies will be quality assessed using a modified Newcastle-0ttawa scale. The review will include narrative synthesis and, if sufficient and comparable data are available, random effects meta-analyses. The review will be conducted in compliance with the Preferred Reporting Items for Systematic Reviews and Meta-Analyses guidelines.

Ethics and dissemination Ethical approval is not required since previously published information from peer-reviewed studies will be assessed. The results of this review will be published in peer-reviewed journals and presented at scientific conferences. Other forms of dissemination will include communication to broader audiences using well-established channels, including through university-based press releases. Progress will be regularly updated on the International Prospective Register of Systematic Reviews to ensure full transparency. PROSPERO registration number CRD42018108881

\section{INTRODUCTION}

In migration and public health research, there has been a growing interest in health risk behaviours, including alcohol consumption, tobacco use, physical inactivity and poor dietary habits. Changes in such behaviours have been identified as a key cause of migrants' health deterioration with longer residence in several contexts (so-called

\section{Strengths and limitations of this study}

- This systematic review will be the first to compile 20 years of evidence on international migrants' health risk behaviours by duration of residence, across multiple country contexts, migrant groups and types of health risk behaviours.

- This review will identify recognisable patterns of behavioural change by duration of residence for specific health behaviours as well as for combinations of them.

- A health equity perspective using the PROGRESSPlus framework will be incorporated into the review to explore inequalities in health risk behaviours beyond migration status.

- The review will additionally compare findings by duration of residence with changes over migrant generations and alternative conceptualisations of behavioural change by acculturation.

- High heterogeneity across studies may entail difficulties for performing and/or interpreting the narrative synthesis and meta-analysis.

unhealthy assimilation). ${ }^{1}$ Although the adoption of unhealthy behaviours among migrants has been extensively studied in the past decades, findings remain scattered across specific migrant groups (eg, Asian Americans $^{2-4}$ and Latin Americans ${ }^{5-8}$ ) and often focus solely on specific health behaviours (eg, smoking, ${ }^{2} 359$ substance use, ${ }^{7}$ alcohol consumption, ${ }^{4} 6{ }^{8}$ physical activity ${ }^{10}$ and $\left.\operatorname{diet}{ }^{11}\right)$. Beyond specific populations and outcomes, limited efforts have been made to comprehensively summarise the existing literature. $^{12}$

Researchers have long been interested in the processes by which migrants become similar (i.e., assimilated) to the native-born population. Migrants' behavioural and lifestyle changes have largely been attributed to acculturation, or the gradual adoption of norms and habits characteristic of the receiving country. ${ }^{13-15}$ Due to difficulties in measuring acculturation, researchers have often come to use duration of residence as a 
proxy among first-generation migrants. However, beyond acting as an indicator of acculturation, duration of residence measures could serve to identify migration stages during which the host context is particularly influential on the adoption of health risk behaviours, which can in turn shape migrants' long-term health. The findings of the proposed review will help to better explain the role of receiving country factors in migrants' health deterioration, and how risk behaviours may be responsible for maintaining (or increasing) avoidable health differences between natives and individuals with a foreign background.

Duration of residence measures could arguably be employed as a public health instrument to increase understanding of migrants' health changes in the receiving context. Considering the social and economic implications of such large-scale health risks ${ }^{16}$ it should be a public health priority to confirm whether migrants generally adopt health risk behaviours with increasing time in the receiving country, and whether this pattern persists across subsequent generations. Furthermore, it is crucial to identify migrant groups that are particularly at risk of adopting negative health behaviours with longer residence based on their countries and regions of origin, that is, due to relevant premigration characteristics. ${ }^{17}$ Together, the findings of the proposed review can help to identify opportunities for effective prevention of migrants' health deterioration over time.

\section{Aims}

To the best of our knowledge, no systematic evaluation of the existing literature on migrants' health risk behaviours by duration of residence has been published to date. This systematic review and meta-analysis will aim:

Aim \#1: To systematically review and synthesise the evidence on changes in health risk behaviours, that is, alcohol consumption, drug and tobacco use, physical inactivity and poor dietary habits, among international adult migrants by duration of residence.

Aim \#2: To identify recognisable patterns of migrant health risk behaviours by duration of residence, and whether these tend to converge with those of the nativeborn population.

Aim \#3: To examine how changes in health risk behaviours extend beyond duration of residence and across migrant generations.

Aim \#4: To determine how changes in health risk behaviours by duration of residence compare to changes by acculturation.

Aim \#5: To identify methodological pitfalls and knowledge gaps in the literature, as well as to provide specific recommendations for future research.

\section{METHODS AND ANALYSIS}

This systematic review will be conducted in compliance with the Preferred Reporting Items for Systematic Reviews and Meta-Analyses (PRISMA) guidelines. ${ }^{18}$
The PRISMA-P (Preferred Reporting Items for Systematic review and Meta-Analysis Protocols) checklist was followed when writing the protocol. ${ }^{19}$

\section{Selection criteria}

Studies will be evaluated and selected according to the criteria outlined below.

\section{Study designs and types of studies}

The systematic review will consider empirical studies utilising quantitative or mixed methods with observational (ie, cross-sectional, cohort, case-control, etc) data. Qualitative and non-observational studies (eg, randomised control trials) will be excluded. Only original articles published in peer-reviewed scientific journals will be assessed, thereby excluding grey literature such as government reports, institutional documents, dissertations (excepting sections published as peer-reviewed articles), books, book chapters, conference abstracts or proceedings, commentaries, blogs, newsletters or any opinion-based publications.

\section{Participants}

This review will assess studies on international migrants, defined by the International Organisation for Migration as 'any person who changes his or her country of usual residence'. ${ }^{20}$ Studies will include migrants across all countries/regions of origin and destination, irrespective of their reason for migration. The review will exclude studies on internal migrants; circular and return migrants; temporary migrants; and minority groups indistinguishable by their foreign-birth status. Only studies examining adults aged 18-64 years at the time of assessment (including individuals who migrated as children) will be included. Migrant groups which are, on average, above or below 18-64 years will be excluded, given that both youth and retirement represent unique life-course transitions that can lead to different lifestyle choices and behavioural changes influenced by factors beyond migration. ${ }^{21-23}$

\section{Exposure}

Studies will be considered if they compare health risk behaviours among foreign-born individuals by duration of residence (ie, time spent in the destination country), including in reference to native-born individuals with or without foreign-born ancestry (ie, second, third or fourthplus generations). From the identified studies, additional information on acculturation instruments based on alternative proxies (eg, language use, cultural orientation, etc) will be considered for first-generation migrants and analysed in relation to duration of residence measures. Studies will be excluded if no measure of duration of residence is included in relation to the outcome of interest.

\section{Outcomes}

Studies will be included if they use measures of one or more of the following health risk behaviours: substance use, including alcohol, drug (ie, illicit substances), and 
tobacco (ie, cigarettes, smokeless tobacco products) use; physical inactivity; and poor dietary habits. The systematic review will not include studies focusing on other types of health risk behaviours (eg, sexual risk behaviours); health outcomes of health risk behaviours (eg, cardiovascular health, related hospitalisations or mortality, etc.); consequences of health risk behaviours (eg, driving under the influence, criminality, etc); nor findings pertaining to attitudes or knowledge regarding health behaviours.

\section{Information sources and search strategy}

The search will be conducted in the PubMed/MEDLINE, Web of Science and ProQuest databases. Only peerreviewed publications written in English and published between 1 January 2000 and 31 December 2019 will be included. Searches will be performed using a basic string template combining search terms related to the population, exposure and outcome components of the research question (eg, 'migrant', 'duration of residence' and 'alcohol', respectively) using Boolean operators (AND, OR and NOT), truncation and wildcard limiters. Based on this template, individual search strings will be created for each database, using controlled vocabulary when appropriate. The search string template has been developed by a project team member with previous experience and expertise in systematic reviews, with input from the rest of the review team members. The search string template is included in online supplemental appendix 1. In addition to the search string, backward and forward citation searching will also be used to identify articles for potential inclusion.

\section{Identification and selection of studies}

One reviewer will conduct the initial search in selected databases and import references into the EndNote X8 reference manager, ${ }^{24}$ removing duplicates and nonrelevant studies by language and publication type. The remaining references will be imported into the Covidence review toolkit. ${ }^{25}$ Two reviewers will independently review and perform a first selection of relevant papers by title and abstract, then by full-length article, based on inclusion criteria. The final decision for inclusion or exclusion of the full-length articles will be taken jointly by these reviewers. Any conflicts in the title/abstract or full-text stages will be resolved in discussions with the other two review team members. Reasons for exclusion will be specified in the final selection of full-text articles. A PRISMA flow chart ${ }^{18}$ will be used to document the full selection process.

\section{Data extraction}

A piloted and standardised data extraction form will be used to extract relevant data from the selected studies (online supplemental appendix 2). Extracted data will include general information (eg, study authors, publication year, country), study characteristics (eg, study design and methodology, sampling characteristics), sample characteristics (eg, sample size, age range, gender proportion, socioeconomic information) and details about exposure and outcome measures and statistical analyses (eg, analytical approach, effect measures, control variables). Data extraction will be done in review pairs, where data are extracted by one reviewer and verified by a second. Disagreements regarding missing or unclear information will be resolved in discussions with the other review team members, or by contacting study authors when necessary.

\section{Quality assessment}

Each included study will be independently and systematically assessed for quality by two researchers, using modified versions of the Newcastle-Ottawa Scale for cross-sectional studies (based on original case-control version) and cohort studies. ${ }^{26} \mathrm{~A}$ third researcher will independently check the assessments for completeness and accuracy. Disparities between quality ratings will be resolved in discussions between all review team members. The quality ratings will be used to guide data analysis and the interpretation of results from the meta-analyses.

\section{Data synthesis}

A summary of descriptive statistics and measures of effect will be provided. Relative measures and associated significance levels of differences in health risk behaviours by exposure will be reported. Data will be synthesised and presented by type of health risk behaviour and population characteristics (eg, sex, country/region of origin and destination).

In order to apply an equity lens to the systematic review, the PROGRESS-Plus framework will also be used to synthesise results according to factors which stratify health inequities beyond migration status (eg, gender, socioeconomic status and age). ${ }^{27}$ The framework has been recommended by the Campbell and Cochrane Equity Methods Group, in relation to the PRISMA-Equity Extension, ${ }^{28}$ to be used when reporting on equity-focused systematic reviews.

\section{Narrative synthesis}

Data will be synthesised using a narrative synthesis approach that follows the Synthesis Without Meta-analysis guidelines $^{29}$ as a complement to the PRISMA guidelines. This checklist was developed to promote transparency and guide clear reporting in systematic reviews using narrative synthesis of quantitative data. The purpose of the narrative synthesis will be to explore patterns of health behaviours based on exposure, especially when meta-analysis is not feasible.

\section{Meta-analysis}

If sufficient and comparable information is available, random effects meta-analyses ${ }^{30}$ will be performed to provide summary estimates by specific health risk behaviours and migrant populations. When necessary, estimates may be transformed to facilitate meta-analysis (eg, by dichotomising estimates for continuous measures or alternating between dichotomous measures). Metaanalysable results will be presented using Forest plots. 
Recommendations from the Meta-analysis of Observational Studies in Epidemiology Group will be followed when reporting results from the meta-analyses. ${ }^{31}$

$\mathrm{I}^{2}$ statistics will be reported to indicate the proportion of variance in meta-analysed estimates attributable to differences in study characteristics (ie, study heterogeneity) ${ }^{32}$ Given sufficient data, subgroup and sensitivity analyses will be performed to examine heterogeneity in the findings by population, exposure and outcome, and to exclude low quality evidence. Meta-analysis will be performed using the metan function in Stata V.13. ${ }^{33}$

Finally, robustness of findings will be assessed using a standardised tool (eg, Risk of Bias in Non-Randomised Studies of Exposures). ${ }^{34}$

\section{Patient and public involvement}

This systematic review will not include any patient and public involvement.

\section{ETHICS AND DISSEMINATION}

Ethical approval is not required since only published information from peer-reviewed studies will be included. The results of this review will be presented at scientific conferences and published in peer-reviewed journals. Other forms of dissemination will include communication to broader audiences using well-established channels, including through university-based press releases. Our progress will be regularly updated on the International Prospective Register of Systematic Reviews to ensure transparency.

\section{AMENDMENTS}

If the published protocol needs to be amended, dates of all amendments, a description of the changes made and the rationale for these changes will be provided in the final systematic review report.

Contributors SPJ and HH conceived the study. LB, N-KG, HH and SPJ planned and designed the study. $\mathrm{HH}$ developed the search strategy with input from all authors. LB and SPJ drafted the manuscript and N-KG and $\mathrm{HH}$ made substantial contributions. All authors approved the final version submitted for publication. LB and SPJ are the guarantors.

Funding This work was supported by the Swedish Research Council for Health, Working Life and Welfare (FORTE), grant number 2016-07128.

Competing interests None declared.

Patient consent for publication Not required.

Provenance and peer review Not commissioned; externally peer reviewed.

Supplemental material This content has been supplied by the author(s). It has not been vetted by BMJ Publishing Group Limited (BMJ) and may not have been peer-reviewed. Any opinions or recommendations discussed are solely those of the author(s) and are not endorsed by BMJ. BMJ disclaims all liability and responsibility arising from any reliance placed on the content. Where the content includes any translated material, BMJ does not warrant the accuracy and reliability of the translations (including but not limited to local regulations, clinical guidelines, terminology, drug names and drug dosages), and is not responsible for any error and/or omissions arising from translation and adaptation or otherwise.

Open access This is an open access article distributed in accordance with the Creative Commons Attribution Non Commercial (CC BY-NC 4.0) license, which permits others to distribute, remix, adapt, build upon this work non-commercially, and license their derivative works on different terms, provided the original work is properly cited, appropriate credit is given, any changes made indicated, and the use is non-commercial. See: http://creativecommons.org/licenses/by-nc/4.0/.

ORCID iD

Lisa Berg http://orcid.org/0000-0002-8707-180X

\section{REFERENCES}

1 Antecol H, Bedard K. Unhealthy assimilation: why do immigrants converge to American health status levels? Demography 2006;43:337-60.

2 Choi S, Rankin S, Stewart A, et al. Effects of acculturation on smoking behavior in Asian Americans: a meta-analysis. J Cardiovasc Nurs 2008;23:67-73.

3 Gotay CC, Reid MS, Dawson MY, et al. Acculturation and smoking in North Americans of Chinese ancestry: a systematic review. Can J Public Health 2015;106:e333-40.

4 Lui PP, Zamboanga BL. Acculturation and alcohol use among Asian Americans: a meta-analytic review. Psychol Addict Behav 2018;32:173-86.

5 Kondo KK, Rossi JS, Schwartz SJ, et al. Acculturation and cigarette smoking in Hispanic women: a meta-analysis. J Ethn Subst Abuse 2016;15:46-72

6 Lui PP, Zamboanga BL. A critical review and meta-analysis of the associations between acculturation and alcohol use outcomes among Hispanic Americans. Alcohol Clin Exp Res 2018;42:1841-62.

7 JY Y, Chen Y, Hussong AM, et al. Acculturation and substance use among Hispanic-Latino and Asian adolescents. Curr Psychiatry Rev 2016;12:139-49.

8 Zemore SE. Acculturation and alcohol among Latino adults in the United States: a comprehensive review. Alcohol Clin Exp Res 2007;31:1968-90.

9 Reiss K, Lehnhardt J, Razum O. Factors associated with smoking in immigrants from non-western to western countries - what role does acculturation play? A systematic review. Tob Induc Dis 2015;13:11.

10 Gerber M, Barker D, Pühse U. Acculturation and physical activity among immigrants: a systematic review. J Public Health 2012;20:313-41.

11 Sanou D, O'Reilly E, Ngnie-Teta I, et al. Acculturation and nutritional health of immigrants in Canada: a scoping review. J Immigr Minor Health 2014:16:24-34.

12 Joshi S, Jatrana S, Paradies Y, et al. Differences in health behaviours between immigrant and non-immigrant groups: a protocol for a systematic review. Syst Rev 2014;3:61.

13 Schwartz SJ, Unger JB, Zamboanga BL, et al. Rethinking the concept of acculturation: implications for theory and research. Am Psychol 2010;65:237-51.

14 Padilla AM, Perez W. Acculturation, social identity, and social cognition: a new perspective. Hisp J Behav Sci 2003;25:35-55.

15 Urquia ML, Gagnon AJ. Glossary: migration and health. J Epidemiol Community Health 2011;65:467-72.

16 Trummer U, Krasnik A. Migrant health: the economic argument. Eur J Public Health 2017;27:590-1.

17 Gushulak BD, MacPherson DW. Health aspects of the pre-departure phase of migration. PLoS Med 2011;8:e1001035.

18 Moher D, Liberati A, Tetzlaff J, et al. Preferred reporting items for systematic reviews and meta-analyses: the PRISMA statement. PLoS Med 2009;6:e1000097.

19 Shamseer L, Moher D, Clarke M, et al. Preferred reporting items for systematic review and meta-analysis protocols (PRISMA-P) 2015: elaboration and explanation. BMJ 2015;350:g7647.

20 International Organization for Migration. Who is a migrant? 2017. Available: www.iom.int/who-is-a-migrant

21 Zantinge EM, van den Berg M, Smit HA, et al. Retirement and a healthy lifestyle: opportunity or pitfall? A narrative review of the literature. Eur J Public Health 2014;24:433-9.

22 Celidoni M, Rebba V. Healthier lifestyles after retirement in Europe? Evidence from SHARE. Eur J Health Econ 2017;18:805-30.

23 Jones SE, Pezzi C, Rodriguez-Lainz A, et al. Health risk behaviors by length of time in the United States among high school students in five sites. J Immigr Minor Health 2016;18:150-60.

24 Clarivate Analytics. EndNote reference management software [computer program] Version X8. Philadelphia, USA: Clarivate Analytics, 2016.

25 Díez E, Peiró R. Interventions to reduce health inequalities [Intervenciones para disminuir las desigualdades en salud].. Gaceta Sanitaria 2004;1:158-67. 
26 Wells G, Shea B, O'Connell D, et al. The Newcastle-Ottawa Scale (NOS) for assessing the quality of nonrandomised studies in metaanalyses, 2009. Available: http://www.ohri.ca/programs/clinical_ epidemiology/oxford.asp

27 O'Neill J, Tabish $\mathrm{H}$, Welch V, et al. Applying an equity lens to interventions: using progress ensures consideration of socially stratifying factors to illuminate inequities in health. J Clin Epidemio 2014;67:56-64.

28 Welch V, Petticrew M, Petkovic J, et al. Extending the PRISMA statement to equity-focused systematic reviews (PRISMA-E 2012): explanation and elaboration. Int J Equity Health 2015;14:92.

29 Campbell M, McKenzie JE, Sowden A, et al. Synthesis without metaanalysis (SWiM) in systematic reviews: reporting guideline. BMJ 2020;368:16890.
30 Borenstein M, Hedges LV, Higgins JPT, et al. A basic introduction to fixed-effect and random-effects models for meta-analysis. Res Synth Methods 2010;1:97-111.

31 Stroup DF, Berlin JA, Morton SC, et al. Meta-Analysis of observational studies in epidemiology: a proposal for reporting. JAMA 2000;283:2008-12.

32 Higgins JPT, Thompson SG, Deeks JJ, et al. Measuring inconsistency in meta-analyses. BMJ 2003;327:557-60.

33 StataCorp. Stata [computer program]. Version 13. College Station, Texas: StataCorp, 2013.

34 Morgan RL, Thayer KA, Santesso N, et al. A risk of bias instrument for non-randomized studies of exposures: a users' guide to its application in the context of GRADE. Environ Int 2019;122:168-84. 